\title{
A contabilização dos ativos por impostos diferidos nos EUA e Europa e o efeito no investidor
}

\section{The accounting of deferred tax assets in the USA and Europe and its effects on the investor}

\author{
Patrícia A. Marrinhas da Costa
}

Instituto Universitário de Lisboa (ISCTE - IUL), Departamento de Contabilidade, Avenida das Forças Armadas, 1649-026 Lisboa, Portugal, patricia_alexandra_costa@iscte.pt

\section{Cláudio A. Figueiredo Pais}

Instituto Universitário de Lisboa (ISCTE - IUL), Escola de Gestão, Departamento de Contabilidade, Investigador da UNID-IUL, Avenida das Forças Armadas, 1649-026 Lisboa, Portugal, claudio.pais@iscte.pt

\section{Resumo}

A contabilização dos ativos por impostos diferidos é diferente, consoante se usem as normas do International Accounting Standards Board (IASB) ou as do Financial Accounting Standards Board (FASB), apesar das diversas tentativas de convergência. Assim, analisamos qual o conjunto de normas, no que respeita aos ativos por impostos diferidos é que tem maior valor relevante para o investidor, como também, quais os ativos por impostos diferidos que têm maior valor relevante, dependendo da sua origem. Tendo por base o modelo de Ohlson (1995), conclui-se que os ativos por impostos diferidos são valor relevante para o investidor. Também se conclui, que a contabilização dos ativos por impostos diferidos usando as normas do FASB, tem maior valor relevante do que usando as normas do IASB. Finalmente conclui-se que os ativos por impostos diferidos decorrentes de diferenças temporárias dedutíveis estão positivamente relacionados com o preço, o que não acontece com os que decorrem de perdas e créditos fiscais, que o estão negativamente.

Palavras-chave: Ativos por impostos diferidos, valor relevante, IASB, FASB.

\begin{abstract}
The accounting of the deferred tax assets is different whether it is used the International Accounting Standards Board (IASB) standards or it is used the Financial Accounting Standards Board (FASB) standards, in spite of all the convergence attempts. Therefore, we analyse which set of accounting standards, regarding the deferred tax assets, is more relevant for the investor, as well, which deferred tax assets are more value relevant, depending on their origin. Based on Ohlson (1995) model, we have concluded that the deferred tax assets are value relevant for the investor. We also have concluded that the accounting of the deferred tax assets using the FASB standards has greater value relevance than using the IASB standards. At least we have concluded that the deferred tax assets arising from deductible temporary differences are positively related with price, unlike the ones arising from losses and credit taxes that are negatively related with the price.
\end{abstract}

Keywords: Deferred tax assets; value relevance, IASB, FASB.

\section{Introdução}

O objetivo deste estudo é verificar qual o conjunto de normas, se do International Accounting Standards Board (IASB) ou do Financial Accounting Standards Board (FASB), na contabilização dos ativos por impostos diferidos, é percebida pelo investidor como sendo a que tem maior valor relevante. Uma quantia contabilística tem valor relevante se tiver uma associação preditiva (significativa) com o valor de mercado do capital próprio, ou seja o preço da ação (Barth, Beaver \& Landsman, 2001). Como as normas do IASB são de aplicação obrigatória na Europa desde 2005 e as do FASB nos Estados Unidos da América (EUA), a contabilização dos impostos diferidos é diferente na Europa e nos EUA e apesar da tentativa de convergência, ainda existem diferenças na contabilização dos impostos diferidos. Em 2009, houve um projeto (Exposure draft (ED) 2009/2) para alterar a norma do IASB dos impostos sobre o rendimento (International Accounting Standard (IAS) 12 Income taxes) e assim diminuir as diferenças entre os dois organismos, o qual foi abandonado.

Os impostos sobre o rendimento, são um tema importante, porque a qualidade de informação pode ser medida através do resultado tributável, sendo utilizado pelos investidores para analisar a possibilidade da empresa estar a manipular os resultados e depende do valor das diferenças entre a contabilidade e a fiscalidade (Hanlon, Kelley \& Shevlin,
2005; Wahab \& Holland, 2012). Segundo Bartov, Goldberg e Kim (2005), Meulen, Gaeremynck e Willekens (2007) e Atwood, Drake, Myers e Myers (2011) a qualidade da informação entre as duas normas (IASB e FASB) parece ser igual porque os investidores não valorizam as diferenças existentes entre as duas normas. Segundo Atwood et al. (2011) a associação entre os fluxos de caixa e os resultados reportados pelas normas do IASB é menor do que para as normas do FASB, contrariamente a McAnally, McGuire e Weaver (2010). Segundo Legoria e Sellers (2005) e Chludek (2011a) os ativos por impostos diferidos são um componente importante para os fluxos de caixa, já que estes tendem a ser mais reversíveis e a permanecer mais tempo nos fluxos de caixa do que os passivos por impostos diferidos, tendo por isso, os primeiros mais relevância para o valor da empresa. Já Lev e Nissim (2004) não encontraram evidência de que os impostos diferidos tenham valor relevante para o investidor, visto não haver relação com a rendibilidade das ações. Segundo Amir e Sougiannis (1999) existe uma relação positiva entre os ativos por impostos diferidos decorrentes de prejuízos fiscais não usados e o preço das ações enquanto Amir, Kirschenheiter e Willard (1997) encontraram uma relação negativa, justificando-a com o facto de os investidores não esperarem que estes sejam recuperados. Phillips, Pincus e Rego (2003), Bauman, Bauman e Halsey (2001) e Christensen, Paik e Stice (2008) encontraram evidência de 
que as empresas usam os impostos diferidos para manipular os resultados e irem ao encontro das previsões dos analistas, o que pode ser conseguido por exemplo através da manipulação da conta de correção do ativo por impostos diferidos (Burgstahler, Elliot \& Hanlon, 2002; Christensen et al., 2008; Frank \& Rego, 2006; Schrand \& Wong, 2003).

Uma motivação para o estudo é a necessidade de convergência entre as normas do IASB e do FASB e de esta ainda não ter sido alcançada. Uma outra motivação é a de que este assunto não ter sido ainda diretamente estudado, apesar de alguns estudos, como por exemplo, os de Meulen et al. (2007), Soderstrom e Sun (2007), Bartov et al. (2005), Atwood et al. (2011), Leuz (2003), Barth, Landsman e Lang (2008) e McAnally et al. (2010) terem comparado as normas. Uma outra motivação tem a ver com importância que os analistas dão aos impostos diferidos, visto estes poderem ser usados para manipular os resultados (Phillips et al., 2003), em particular por os ativos por impostos diferidos influenciarem os fluxos de caixa (Legoria \& Sellers, 2005; Chludek, 2011b) e poderem ser materiais. Segundo Poterba, Rao e Seidman (2011) estes representavam em termos líquidos, cerca de 5 por cento do total do ativo de 35 por cento das empresas da amostra e que para 10 por cento das empresas estes representavam 10 por cento do total de ativo. Assim, pretendemos contribuir em termos da convergência entre as normas do IASB e do FASB e auxiliálos em saber em que áreas dos ativos por impostos diferidos deverão prestar mais atenção.

Concluímos que há evidências de que os ativos por impostos diferidos têm valor relevante para o investidor, corroborando Legoria e Sellers (2005) e Chludek (2011b) mas contrariando Lev e Nissim (2004), como também, que a contabilização dos ativos por impostos diferidos usando as normas do FASB, estão mais associados com o preço do que usando as normas do IASB, visto existirem mais restrições para o seu reconhecimento. Esta conclusão é contrária à de McAnally et al. (2010), porque se os ativos por impostos diferidos forem contabilizados pelas normas do IASB, estes predizem melhor o valor futuro dos fluxos de caixa, mas confirma as conclusões de Atwood et al. (2011). Também há evidência, de que os ativos por impostos diferidos de diferenças temporárias dedutíveis têm maior valor relevante do que os ativos por impostos diferidos de perdas e créditos fiscais não usados.

Após esta introdução o estudo está organizado da seguinte forma. Na secção 2 apresenta-se uma revisão das normas dos impostos diferidos do IASB e do FASB. Na secção 3 apresenta-se a revisão de literatura relacionada. Na secção 4 é apresentado o desenho de investigação. Na secção 5 descreve-se a amostra, a estatística descritiva e apresentam-se os resultados. Finalmente na secção 6 apresenta-se a conclusão.

\section{Análise das normas}

A norma do IASB é a IAS 12 enquanto a do FASB é o código Accounting Standard Codification (ASC) 740. Em termos de princípios genéricos, não há diferenças entre as normas do IABS e do FASB. Os passivos por impostos diferidos resultam de diferenças temporárias tributáveis e os ativos por impostos diferidos resultam de diferenças temporárias dedutíveis e de perdas e créditos fiscais não usados. 0 critério genérico de reconhecimento dos ativos e dos passivos por impostos diferidos é o total e a mensuração é na base das leis e taxas fiscais aprovadas à data do balanço. Para a IAS 12 os ativos por impostos diferidos só devem ser reconhecidos se for provável a existência de lucro tributável nos períodos em que as diferenças temporárias dedutíveis reverterem e as perdas e créditos fiscais sejam usados. Para a ASC 740 os ativos por impostos diferidos são todos reconhecidos, sendo depois reduzidos por uma conta de correção, se existir mais de 50 por cento de probabilidade de não os recuperar. Contudo, de acordo com a IAS 12, não se devem reconhecer impostos diferidos de diferenças temporárias resultantes do reconhecimento inicial do goodwill e de ativos ou passivos que não sejam resultantes de concentrações empresariais ou de transações que não afetem o resultado contabilístico. Ainda segundo a IAS 12 e no caso de perdas e créditos fiscais não usados, como estes são uma forte da probabilidade de inexistência de lucros tributáveis futuros, se a empresa tiver uma história de prejuízos recentes, os ativos por impostos diferidos só devem ser reconhecidos até ao valor das diferenças temporárias tributáveis que revertam nos mesmos períodos ou se houver outras provas convincentes da existência de lucros tributáveis futuros. Estas limitações não existem de acordo com a ASC 740, o que existe é um julgamento com base na probabilidade de 50 por cento, para corrigir os ativos por impostos diferidos já reconhecidos.

\section{Revisão da literatura}

O estudo dos impostos diferidos e a análise da informação desses impostos são elementos importantes na estimativa de resultados e estes no valor da empresa (Amir \& Sougiannis, 1999). O investidor utiliza o lucro tributável para análise da possibilidade da empresa estar a fazer a gestão de resultados, condicionado assim a qualidade da informação, o que indica que o lucro tributável e que as diferenças entre as normas da contabilidade e as regras fiscais, proporcionam informação adicional útil para os investidores (Hanlon et al., 2005; Hanlon, 2005; Wahab \& Holland, 2012).

As normas do IASB são baseadas em princípios e as do FASB em regras (Meulen et al., 2007) e podem influenciar a qualidade da informação. Contudo Bartov et al. (2005), Meulen et al. (2007) e Atwood et al. (2011) encontraram evidências de que o valor relevante dos resultados pelas normas do IASB e do FASB não é diferente. Usando as normas do IASB o valor de mercado das empresas é maior (Ashbaugh \& Pincus, 2001; Grossman, Smith \& Tervo, 2013), mas Leuz (2003) não encontrou qualquer evidência de que a qualidade da informação pelas normas do IASB seja melhor do que pelas normas do FASB. Quanto aos ativos por impostos diferidos, McAnally et al. (2010) encontraram evidências de que se contabilizados usando as normas do IASB, são melhores a predizerem os fluxos de caixa futuros, do que se o forem pelas normas do FASB.

De acordo com Chludek (2011a) os investidores na avaliação da empresa não incluem os impostos diferidos, a 
não ser que o seu valor seja elevado. De acordo com Amir, Kirschenheiter e Willard (2001) os impostos diferidos afetam o valor da empresa. Segundo Chludek (2011a) os ativos por impostos diferidos têm maior relevância para o valor da empresa dos que os passivos por impostos diferidos. Legoria e Sellers (2005) encontraram evidências de que a contabilização dos impostos diferidos de acordo com a norma do FASB dá informações relevantes aos utilizadores, tendo concluído que existia uma relação positiva entre os ativos por impostos diferidos e os fluxos de caixa operacionais futuros, o que é corroborado por Chludek (2011b), mas não por Lev e Nissim (2004).

A gestão dos resultados através da conta de correção dos ativos por impostos diferidos, é feita reconhecendo um valor mais elevado do que o correto, reduzindo o resultado do período corrente e assim aumentando o de períodos futuros (Christensen et al., 2008; Burgstahler et al., 2002; Frank \& Rego, 2006). Amir e Sougiannis (1999) concluíram que os investidores encaram os ativos por impostos diferidos como uma redução de pagamento de impostos no futuro e por isso, encontraram uma associação forte com o preço, não sendo então vistos como um prenúncio de mais perdas fiscais, que foi a evidência encontrada por Amir et al. (1997).

\section{Desenho da investigação}

Os impostos diferidos são importantes, visto fazerem parte do imposto sobre o rendimento, terem uma associação forte com o preço (Amir \& Sougiannis, 1999; Amir et al., 1997) e serem uma parte material do ativo (Poterba et al., 2011). Também os ativos por impostos diferidos são materiais no ativo das empresas (Chludek, 2011a) e existe evidência de uma associação com os fluxos de caixa futuros (Legoria \& Sellers, 2005; Chludek, 2011a; Chludek, 2011b), apesar de Lev e Nissim (2004) não terem encontrado evidência de associação entre os ativos por impostos diferidos e a rendibilidade das ações. Em virtude dos resultados contraditórios, surge a primeira hipótese de investigação, que relaciona o preço das ações com o valor dos ativos por impostos diferidos:

H1: Os ativos por impostos diferidos têm valor relevante para o investidor.

Como a contabilização dos ativos por impostos diferidos não é igual entre as normas do IASB e as normas do FASB, apesar de existirem tentativas para convergir as normas do IASB com as do FASB; como os estudos são contraditórios sob a sua utilidade; e como a relação, no caso especifico dos ativos por impostos diferidos, entre as normas do IASB e do FASB não ter sido estudado, na perspetiva do valor relevante para o investidor; temos então a segunda hipótese, que relaciona o preço com os ativos por impostos diferidos, tentando encontrar evidência de qual a norma (IASB ou FASB) tem maior valor relevante para o investidor:

H2: Os ativos por impostos diferidos têm mais valor relevante para o investidor, usando as normas do FASB, do que usando as normas do IASB.

Apesar de vários estudos terem analisado as diferenças temporárias (perdas e créditos fiscais não usados) que dão origem a ativos e a passivos por impostos diferidos (Amir et al., 1997; Amir \& Sougiannis, 1999; Guenther \& Sansing, 2000; Laux, 2013; Chludek, 2011a), sendo as conclusões nalguns casos contraditórias, não existe nenhum estudo que tenha feito a análise em função da comparação entre as normas do IASB e do FASB e por isso surge a terceira hipótese, que relaciona o preço com os ativos por impostos diferidos separados em função do que lhe deu origem (diferenças temporárias dedutíveis e perdas e créditos fiscais não usados):

H3: Os ativos por impostos diferidos têm valor relevante diferente para o investidor, independentemente de terem resultado de diferenças temporárias dedutíveis ou de perdas e créditos fiscais não usados.

Os modelos dos preços são frequentemente usados para providenciar conhecimento acerca do valor relevante de uma informação contabilística especifica para os organismos de normalização (Barth et al., 2001). Usando o preço das ações como critério sumário de mensuração da informação que é considerada pelo investidor como relevante e investigando a capacidade das quantias contabilísticas para explicar essa mensuração temos a equação (1), que relaciona o preço das ações e o ativo por impostos diferidos. Esta equação e as posteriores (1 a 3) podem ser vistas como baseadas no modelo de avaliação de Ohlson (1995), que fornece uma relação formal entre avaliação e dados contabilísticos, sendo uma extensão do modelo de Preinreich (Dumontier \& Raffournier, 2002). Por este modelo o valor de mercado do capital próprio é igual ao seu valor contabilístico ajustado por ganhos correntes mensurados pelos lucros anormais e outras informações que modifiquem a previsão dos ganhos futuros.

$$
P_{i t}=\beta_{0}+\beta_{1} A_{i t}+\beta_{2} L_{i t}+\beta_{3} N_{i t}+\beta_{4} D T A_{i t}+\varepsilon_{i t}
$$

Onde o $P$ é o preço por ação a 31 de dezembro; $\beta_{0}, \beta_{1}, \beta_{2}, \beta_{3} e$ $\beta_{4}$ são os coeficientes das variáveis independentes; $A$ é o total do ativo líquido dos ativos por impostos diferidos; $L$ é o total do passivo; NI é o resultado líquido; e DTA são os ativos por impostos diferidos. Todas as variáveis são divididas pelo número de ações. Estima-se que o coeficiente das variáveis $A, N I$ e DTA tenha sinal positivo e que o coeficiente da variável $L$ tenha sinal negativo. Se o coeficiente da variável DTA tiver sinal positivo (como se prevê), significa que os ativos por impostos diferidos têm valor relevante. 0 uso do preço por ação é para diminuir a possibilidade da existência de problemas de heterocedasticidade. A estandardização das variáveis independentes (divisão pelo número de ações) é para evitar os efeitos de escala porque eles podem afetar as inferências. Os efeitos de escala nas inferências de estudos de valor relevante baseados nos coeficientes de equações de corte seccional são uma preocupação. São excluídas as observações em que o capital próprio seja negativo.

A segunda equação (2), que continua a relacionar o preço com dados contabilísticos (ativo líquido dos ativos por impostos diferidos, passivo, resultado líquido e ativos por 
impostos diferidos), inclui uma variável dummy para análise de qual o conjunto de normas, se do IASB, se do FASB, tem

maior valor relevante para o investidor, ou seja, que tenham um maior grau de associação com o preço.

Onde $A C O$ é uma variável dummy que assume o valor 1 para empresas que usem as normas do IASB (Europa) e 0 para empresas que usem as normas do FASB (EUA) e ACO $\times$ DTA é uma variável de teste à interação entre a variável dummy e os ativos por impostos diferidos. É através do sinal do coeficiente desta última variável, que se for positivo, se conclui que as normas do IASB têm maior valor relevante

$$
P_{i t}=\beta_{0}+\beta_{1} A_{i t}+\beta_{2} L_{i t}+\beta_{3} N I_{i t}+\beta_{4} T D_{i t}+\beta_{5} L C_{i t}+\beta_{6} V A_{i t}+\varepsilon_{i t}
$$

A diferença entre a primeira equação e esta última (3), é a divisão da variável ativos por impostos diferidos (DTA) em três novas variáveis. A variável $T D$ que é de diferenças temporárias dedutíveis, a variável $L C$ que é de perdas e créditos fiscais não usados, e por fim a variável $V A$ que é da conta de correção. Espera-se que o coeficiente das variáveis $T D$ e $L C$ seja positivo e que o sinal do coeficiente da variável $V A$ seja negativo. Não se consegue estimar é qual a variável que terá maior valor relevante, porque no caso das diferenças temporárias dedutíveis, existe alguma incerteza do momento em que irão ser revertidas e relativamente às perdas e créditos fiscais não usados, podem ser considerados como reduções de pagamentos de impostos no futuro mas também como um prenúncio de mais perdas fiscais.

\section{Amostra e resultados}

5.1 Amostra Partindo das maiores 150 empresas do Stoxx Europe 600 (Europa) e das maiores 150 empresas do índice Standards \& Poors (S\&P) 500 (EUA) e tendo por para o investidor, mas no caso em que seja negativo, já são as normas do FASB que têm maior valor relevante para o investidor.

A associação dos diferentes tipos de ativos por impostos por impostos diferidos (se de diferenças temporárias dedutíveis ou de perdas e créditos fiscais) com o preço é analisada na terceira e última equação (3).

base informação à data de fecho de 2007 a 2012, excluíram-se as empresas que não tivessem valores de ativos por impostos diferidos, empresas que tivessem um período contabilístico diferente do ano civil, empresas financeiras (por causa dos requisitos de relato diferentes) e empresas que usassem normas diferentes do que é suposto usarem. Os dados da contabilidade e de mercado são obtidos da DataStream Wordscope Global Database (DataStream), excluindo os ativos por impostos diferidos brutos e a sua composição (de diferenças temporárias dedutíveis e de perdas e créditos fiscais não usados), que são obtidos diretamente dos relatórios financeiros (notas). A construção da amostra é a da Tabela 1. Partindo de uma amostra inicial de 300 empresa foram eliminadas 141 empresas, das quais 27 empresas por falta de dados, 62 empresas por terem um período contabilístico diferente do ano civil, 51 empresas por serem empresas financeiras e 1 empresa por usar normas diferentes do esperado face à região. A amostra final corresponde a 159 empresas e a 927 observações.

Tabela 1 - Definição da amostra

\begin{tabular}{|l|c|c|c|c|c|c|}
\hline & \multicolumn{2}{|c|}{ Global } & \multicolumn{2}{c|}{ Europa } & \multicolumn{2}{c|}{ EUA } \\
\hline & Número & $\%$ & Número & $\%$ & Número & $\%$ \\
\hline Amostra inicial & 300 & 100,0 & 150 & 100,0 & 150 & 100,0 \\
\hline Empresas retiradas: & & & & & & \\
\hline Empresas com falta de dados & -27 & 9,0 & -5 & 3,3 & -22 & 14,7 \\
\hline Empresas com período contabilístico diferente & -62 & 20,7 & -20 & 13,3 & -42 & 28,0 \\
\hline Empresas financeiras & -51 & 17,0 & -37 & 24,7 & -14 & 9,3 \\
\hline Empresas com normas diferentes & -1 & 0,3 & -1 & 0,7 & 0 & 0,0 \\
\hline Amostra final & 159 & 53,0 & 87 & 58,0 & 72 & 48,0 \\
\hline
\end{tabular}

\subsection{Análise descritiva}

Na Tabela $\mathbf{2}$ apresentam-se as estatísticas descritivas de todas as variáveis e de todas as equações (1 a 3), separadas por empresas da Europa e dos EUA. A média (mediana) dos ativos por impostos diferidos totais por ação é de 2,58 $(1,44)$, correspondendo a 5,62 $(4,51)$ por cento da média (mediana) dos ativos por ação (excluem os ativos por impostos diferidos). A média (mediana) dos ativos por impostos diferidos por ação da Europa $(2,59(1,25))$ é maior que a das empresas dos EUA $(2,56(1,6))$, contudo a média (mediana) dos ativos por impostos diferidos dos EUA corresponde a $7,42(5,45)$ por cento dos ativos por ação dos EUA, mais do que na Europa $(4,73(3,53)$ por cento dos ativos por ação). Quanto à decomposição dos ativos por impostos diferidos, a média (mediana) dos ativos por impostos diferidos das diferenças temporárias dedutíveis é de 2,08 (1,16), correspondendo a $80,62(80,56)$ por cento dos ativos por impostos diferidos por ação, dos quais $2,2(1,37)$ são de empresas dos EUA e 1,99 $(0,98)$ são de empresas da Europa. A média (mediana) dos ativos por impostos diferidos de perdas e créditos fiscais não usados por ação é de $0,74(0,24)$, correspondendo a $28,68(16,67)$ por cento dos ativos por impostos diferidos por ação, dos quais $0,91(0,31)$ são de empresas dos EUA e 0,61 $(0,15)$ são de empresas da Europa. A média (mediana) da redução do ativo por impostos diferidos é de 0,24 (0) que corresponde a 9,30 (0) por cento dos ativos por impostos diferidos, dos quais 0,55 $(0,1)$ são de empresas dos EUA e das empresas da Europa são 0 (0), visto não usarem essa conta pelas normas do IASB. As empresas da amostra são lucrativas (a média (mediana) dos resultados por ação é de € 2,61 (€ 1,86). 
Tabela 2 - Estatística descritiva

\begin{tabular}{|c|c|c|c|c|c|c|c|c|c|c|c|c|}
\hline \multirow[b]{2}{*}{ Variáveis } & \multicolumn{3}{|c|}{ Média } & \multicolumn{3}{|c|}{ Mediana } & \multicolumn{3}{|c|}{ Desvio padrão } & \multicolumn{3}{|c|}{$\boldsymbol{N}$} \\
\hline & Europa & $E U A$ & Total & Europa & $E U A$ & Total & Europa & $E U A$ & Total & Europa & $E U A$ & Total \\
\hline $\mathrm{P}$ & 41,27 & 48,62 & 44,51 & 27,54 & 36,92 & 32,6 & 49,28 & 62,59 & 55,62 & 519 & 408 & 927 \\
\hline A & 54,81 & 34,49 & 45,87 & 35,41 & 29,34 & 31,95 & 54,66 & 25,92 & 45,48 & 519 & 408 & 927 \\
\hline $\mathrm{L}$ & 36,79 & 22,37 & 30,44 & 21,02 & 16,49 & 18,95 & 41,42 & 19,49 & 34,32 & 519 & 408 & 927 \\
\hline NI & 2,63 & 2,58 & 2,61 & 1,78 & 2,03 & 1,86 & 3,67 & 3,14 & 3,44 & 519 & 408 & 927 \\
\hline DTA & 2,59 & 2,56 & 2,58 & 1,25 & 1,6 & 1,44 & 3,29 & 3,015 & 3,17 & 519 & 408 & 927 \\
\hline ACO & 1 & 0 & 0,56 & 1 & 0 & 1 & 1 & 0 & 0,5 & 519 & 408 & 927 \\
\hline ACO $\times$ DTA & 2,59 & 0 & 1,45 & 1,25 & 0 & 0,24 & 3,29 & 0 & 2,78 & 519 & 408 & 927 \\
\hline TD & 1,99 & 2,2 & 2,08 & 0,98 & 1,37 & 1,16 & 2,61 & 2,75 & 2,67 & 519 & 408 & 927 \\
\hline LC & 0,61 & 0,91 & 0,74 & 0,15 & 0,31 & 0,24 & 1,4 & 2,14 & 1,77 & 519 & 408 & 927 \\
\hline VA & 0 & 0,55 & 0,24 & 0 & 0,1 & 0 & 0 & 1,81 & 1,23 & 519 & 408 & 927 \\
\hline
\end{tabular}

\subsection{Resultados dos testes das regressões}

A Tabela 3 mostra as estatísticas relativas à equação (1). A regressão (bem como as posteriores) inclui um conjunto de variáveis dummy para cada período, de forma a poder reduzir os efeitos macroeconómicos específicos de cada período (que é o caso da crise financeira de 2008), assumindo o valor de 1 , se a observação é do período em análise e 0 se não o for. São também eliminados os outliers (66 observações), tendo sido calculados com base da distância Cook para a variável dependente e os resíduos estandardizados para as variáveis independentes, o que foi feito também para as equações seguintes. Os resultados de todos os testes apresentados baseiam-se numa regressão que utiliza variâncias de coeficientes que são válidas mesmo havendo heterocedasticidade pelo teste White (Johnston \& DiNardo, 2000). Tal como previsto, os ativos (líquidos dos ativos por impostos diferidos) estão relacionados positivamente com o preço, tal como os resultados líquidos, enquanto os passivos estão negativamente relacionados com o preço, sendo estatisticamente significativos a um nível de significância de 1 por cento. Quanto aos ativos por impostos diferidos estão positivamente relacionados com o preço a um nível de significância de 1 por cento, o que significa que para os investidores, estes ativos têm valor relevante e como o coeficiente é de 0,93 , significa que o preço aumenta 0,93 face a um aumento de 1 dos ativos por impostos diferidos.

Tabela 3 - Valor relevante dos ativos por impostos diferidos

\begin{tabular}{|l|c|c|c|c|}
\hline \multicolumn{1}{|c|}{ Variáveis } & Predição & Coeficiente & \multicolumn{2}{c|}{ Estatística-t } \\
\hline Constante & & 10,531 & 6,443 & $0,000^{* * *}$ \\
\hline A & + & 0,609 & 10,048 & $0,000^{* * *}$ \\
\hline L & - & $-0,729$ & $-8,559$ & $0,000^{* * *}$ \\
\hline NI & + & 9,233 & 19,625 & $0,000^{* * *}$ \\
\hline DTA & + & 0,930 & 3,581 & $0,000^{* * *}$ \\
\hline N & 861 & & & \\
\hline $\mathrm{R}^{2}$ ajustado & 0,735 & & & \\
\hline Estatística $\mathrm{F}$ & $266,0189^{* * *}$ & & & \\
\hline$* * *$ & & & \\
\hline
\end{tabular}

Para verificar qual o conjunto de normas (do IASB ou do FASB) tem maior valor relevante na contabilização dos ativos por impostos diferidos, foi corrida a regressão da equação (2) e os resultados são os da Tabela 4. Todos os coeficientes são estatisticamente significativos a um nível de 1 por cento, tendo o sinal estimado, excluindo o coeficiente da variável de interesse $(A C O \times D T A)$, que apesar de ter o sinal esperado (negativo), significando que os ativos por impostos diferidos têm menor valor relevante se forem contabilizados usando as normas do IASB, não se pode rejeitar a hipótese nula do coeficiente ser nulo.

Tabela 4 - Valor relevante das normas na contabilização dos ativos por impostos diferidos

\begin{tabular}{|l|c|c|c|c|}
\hline \multicolumn{1}{|c|}{ Variáveis } & Predição & Coeficiente & \multicolumn{2}{c|}{ Estatística-t } \\
\hline Constante & & 13,887 & 7,443 & $0,000^{* * *}$ \\
\hline A & + & 0,699 & 9,133 & $0,000^{* * *}$ \\
\hline L & - & $-0,806$ & $-7,527$ & $0,000^{* * *}$ \\
\hline NI & + & 9,230 & 18,848 & $0,000^{* * *}$ \\
\hline DTA & + & 0,930 & 2,628 & $0,009 * * *$ \\
\hline ACO & - & $-5,825$ & $-4,638$ & $0,000^{* * *}$ \\
\hline ACO $*$ DTA & - & $-0,258$ & $-0,572$ & 0,5675 \\
\hline N & 868 & & & \\
\hline $\mathrm{R}^{2}$ ajustado & 0,780 & & & \\
\hline Estatística F & $280,619 * * *$ & & & \\
\hline$* * *$ & & & \\
\end{tabular}


A Tabela 5 apresenta as estatísticas da equação (3). A regressão inclui uma variável dummy para controlo do conjunto de normas usadas (visto a conta de correção dos ativos por impostos diferidos só existir pelas normas do FASB). Todos os coeficientes das variáveis comuns às três equações têm o sinal estimado e estatisticamente significativo a um nível de 1 por cento. 0 coeficiente da variável ativo por impostos diferidos de diferenças temporárias dedutíveis (TD) é positivo enquanto o da variável ativo por impostos diferidos de perdas e créditos fiscais não usados $(L C)$ é negativo, significando que existe uma associação positiva com o preço (este aumenta) no primeiro caso e negativa no segundo caso, contudo não se pode rejeitar a hipótese nula do coeficiente ser nulo, no caso da variável $L C$. Assim, pode-se concluir que o investidor parte do pressuposto que os ativos por impostos diferidos de perdas e créditos fiscais não são recuperáveis no futuro, porque a expetativa é de que se vão criar mais perdas fiscais no futuro, ou então o período para os recuperar é demasiado pequeno, o que confirma as conclusões de Amir et al. (1997). Tal como previsto, a variável correção do ativo por impostos diferidos (VA) tem uma associação negativa com o preço, contudo não se pode rejeitar a hipótese nula do coeficiente ser nulo.

Tabela 5 - Valor relevante dos ativos por impostos diferidos de diferenças temporárias dedutíveis e de perdas e créditos fiscais não usados

\begin{tabular}{|l|c|c|c|c|}
\hline \multicolumn{1}{|c|}{ Variáveis } & Predição & Coeficiente & \multicolumn{2}{|c|}{ Estatística-t } \\
\hline Constante & & 18,880 & 12,620 & $0,000^{* * *}$ \\
\hline A & + & 0,802 & 13,423 & $0,000^{* * *}$ \\
\hline L & - & $-0,882$ & $-12,025$ & $0,000^{* * *}$ \\
\hline NI & + & 7,122 & 20,144 & $0,000^{* * *}$ \\
\hline TD & + & 0,873 & 3,024 & $0,003^{* * *}$ \\
\hline LC & + & $-0,129$ & $-0,191$ & 0,849 \\
\hline VA & - & $-0,134$ & $-0,111$ & 0,911 \\
\hline ACO & - & $-7,654$ & & \\
\hline N & 853 & & & \\
\hline R ajustado & 0,771 & & & \\
\hline Estatística $\mathrm{F}$ & $269,166^{* * *}$ & & & \\
\hline$* * *$ & & & \\
\hline
\end{tabular}

Correndo a regressão anterior (3), mas separando as empresas em função das normas usadas (IASB ou FASB, ou seja, Europa ou EUA), os resultados são os da Tabela 6. Os resultados na Europa são similares aos resultados da Tabela 5. No caso dos EUA (normas do FASB), o sinal do coeficiente das variáveis $T D$ e $L C$ é positivo, apesar deste último não ser estatisticamente significativo a um nível de significância de 10 por cento. Assim, isto quer dizer que ambos são valor relevante para o investidor, por isso o teste do Wald para os comparar e como o $p$-value é 0 , implica rejeitar a hipótese nula de que os coeficientes sejam iguais, pelo que, os investidores atribuem maior valor relevante aos ativos por impostos diferidos das perdas e créditos fiscais, o que pode ser justificado pelos períodos de reporte das perdas fiscais (mais períodos para a frente) e o que confirma Amir e Sougiannis (1999).

Tabela 6 - Valor relevante dos ativos por impostos diferidos de diferenças temporárias dedutíveis e de perdas e créditos fiscais não usados para a Europa e EUA

\begin{tabular}{|c|c|c|c|c|c|c|c|}
\hline \multirow{3}{*}{$\begin{array}{l}\text { Variáveis } \\
\text { Constante }\end{array}$} & \multirow{3}{*}{ Predição } & \multicolumn{2}{|c|}{ Coeficiente } & \multicolumn{4}{|c|}{ Estatística-t } \\
\hline & & \multirow{2}{*}{$\begin{array}{c}\text { Europa } \\
3,855\end{array}$} & \multirow{2}{*}{$\begin{array}{c}\boldsymbol{E} \boldsymbol{U A} \\
12,375\end{array}$} & \multicolumn{2}{|c|}{ Europa } & \multicolumn{2}{|c|}{$E U A$} \\
\hline & & & & 2,341 & $0,020^{* *}$ & 5,555 & $0,000^{* * *}$ \\
\hline A & + & 0,770 & 0,551 & 12,719 & $0,000^{* * *}$ & 5,135 & $0,000^{* * *}$ \\
\hline $\mathrm{L}$ & - & $-0,865$ & $-0,642$ & $-11,385$ & $0,000^{* * *}$ & $-5,090$ & $0,000^{* * *}$ \\
\hline NI & + & 8,358 & 8,149 & 23,255 & $0,000^{* * *}$ & 14,115 & $0,000^{* * *}$ \\
\hline TD & + & 1,413 & 0,545 & 3,918 & $0,000^{* * *}$ & 1,329 & 0,185 \\
\hline LC & + & $-3,317$ & 5,405 & $-4,077$ & $0,000^{* * *}$ & 6,658 & $0,000^{* * *}$ \\
\hline VA & - & & $-4,242$ & & & $-5,216$ & $0,000^{* * *}$ \\
\hline $\mathrm{N}$ & & 480 & 366 & & & & \\
\hline $\mathrm{R}^{2}$ ajustado & & 0,825 & 0,601 & & & & \\
\hline Estatística F & & $226,974^{* * *}$ & $51,002^{* * *}$ & & & & \\
\hline \multicolumn{8}{|l|}{ Teste Wald } \\
\hline Restrição & & & & & & Estatística-t & \\
\hline$B_{4}=\beta_{5}$ & & & & & & 31,219 & $0,000^{* * *}$ \\
\hline
\end{tabular}

\section{Conclusões}

Os ativos por impostos diferidos são uma parte material do valor dos ativos das empresas e os critérios de reconhecimento e de mensuração podem afetar a perceção do seu valor por parte dos investidores. Por isso o estudo do valor relevante dos ativos por impostos diferidos quanto à norma usada na sua contabilização e quanto ao que os origina.

Os resultados confirmam a expetativa, de que os ativos por impostos diferidos são relevantes para o investidor, de que 
os ativos por impostos diferidos calculados com base nas normas do FASB têm maior valor relevante do que contabilizados com base nas normas do IASB, visto haver menos restrições no seu reconhecimento e de que os ativos por impostos diferidos decorrentes de diferenças temporárias dedutíveis estão positivamente associados com o preço, o que não acontece com os decorrentes de perdas e créditos fiscais não usados.

Uma limitação do estudo foi de o valor bruto dos ativos por impostos diferidos não estar disponível na DataStraem, por isso foi obtido diretamente dos relatórios financeiros e uma outra foi a impossibilidade de separar os ativos por impostos diferidos de perdas fiscais e de créditos fiscais não usados.

Como trabalhos futuros, sugere-se a replicação para empresas não cotadas e de menor dimensão, como também separar os ativos por impostos diferidos das diferentes diferenças temporárias dedutíveis.

\section{Referências}

Amir, E., \& Sougiannis, T. (1999). Analysts' interpretation and investors' valuation of tax carryforwards. Contemporary Accounting Research, 16(1), 1-33.

Amir, E., Kirschenheiter, M., \& Willard, K. (1997). The valuation of deferred taxes. Contemporary Accounting Research, 14(4), 597-622.

Amir, E., Kirschenheiter, M., \& Willard, K. (2001). The aggregation and valuation of deferred taxes. Review of Accounting Studies, 6(23), 275-297.

Ashbaugh, H., \& Pincus, M. (2001). Domestic accounting standards, international accounting standards, and the predictability of earnings. Journal of Accounting Research, 39(3), 417-434.

Atwood, T., Drake, M., Myers, J., \& Myers, L. (2011). Do earnings reported under IFRS tell us more about future earnings and cash flows? Journal of Accounting and Public Policy, 30, 103-121.

Barth, M., Beaver, W., \& Landsman, W. R. (2001). The relevance of the value relevance literature for financial accounting standard setting: another view. Journal of Accounting and Economics, 31, 77-104.

Barth, M., Landsman, W., \& Lang, M. (2008). International accounting standards and accounting quality. Journal of Accounting Research, 46(3), 467-497.

Bartov, E., Goldberg, S., \& Kim, M. (2005). Comparative value relevance among German, U.S. and international accounting standards: a German stock market perspective. Journal of Accounting, Auditing \& Finance, 20(2), 95-119.

Bauman, C., Bauman, M., \& Halsey, R. (2001). Do firms use the deferred tax asset valuation allowance to manage earnings? Journal of the American Taxation Association ${ }_{\mathbf{L}} 23(2), 27-48$.

Burgstahler, D., Elliot, W., \& Hanlon, M. (2002). How firms avoid losses: evidence of use of the net deferred tax asset account. Working paper. University of Michigan Business School.

Chludek, A. (2011a). Perceived versus actual cash flows implications of deferred taxes: an analysis of value relevance and reversal under IFRS. Journal of International Accounting Research, 10(1), 1-25.

Chludek, A. (2011b). On the relation of deferred taxes and cash flows. Working paper. Cologne Graduate School in Management, Economics and Social Sciences, University of Cologne.

Christensen, T., Paik, G., \& Stice, E., (2008). Creating a bigger bath using the deferred tax valuation allowance. Journal of Business Finance \& Accounting, 35, 601-625.

Dumontier, P., \& Raffoutnier, B. (2002). Accounting and Capital Markets: A Survey of the European Evidence. The European Accounting Review, 11, 119-151.

Financial Accounting Standards Board (FASB). Accounting Standard Codification 740: Income Taxes, Norwalk (CT).
Frank, M., \& Rego. S. (2006). Do managers use the valuation allowance account to manage earnings around certain earnings targets? Journal of the American Taxation Association, 28(1), 43-65.

Grossman, A., Smith, L., \& Tervo, W. (2013). Measuring the impact of international reporting standards on market performance of publicly traded companies. Advances in Accounting, incorporating Advances in International Accounting, 29, 343-349.

Guenther, D., \& Sansing, R. (2000). Valuation of the firm in the presence of temporary book tax differences: the role of deferred tax assets and liabilities. The Accounting Review, 75(1), 1-12.

Hanlon, M. (2005). The persistence and pricing of earnings, accruals, and cash flows when firms have large book-tax differences. The Accounting Review, 80(1), 137-166.

Hanlon. M., Kelley, S., \& Shevlin, T. (2005). Evidence on the possible information loss of conforming book income and taxable income. Journal of Law \& Economics, 48(2), 407-442.

International Accounting Standards Board (IASB) (2001). International Accounting Standard 12: Income taxes, London.

Johnston, J., \& DiNardo, J. (2000). Econometric Methods. Econometric Theory, 16, 139-142.

Laux, R. (2013). The association between deferred tax assets and liabilities and future tax payments. The Accounting Review, 88(4), 1357-1383.

Legoria, J., \& Sellers, K. (2005). The analysis of SFAS No. 109's usefulness in predicting future cash flows from a conceptual framework perspective. Research in Accounting Regulation, 18,143-161.

Leuz, C. (2003). IAS versus U.S.GAAP: information asymmetrybased evidence from Germany's new market. Journal of Accounting Research, 41(3), 445-471.

Lev, B., \& Nissim, D. (2004). Taxable income, future earnings and equity values. The Accounting Review, 79(4), 1039-1074.

McAnally, M., McGuire, S., \& Weaver, C. (2010). Assessing the financial reporting consequences of conversion to IFRS: the case of equity-based compensation. Accounting Horizons, 24(4), 589-621.

Meulen, S., Gaeremynck, A., \& Willekens, M. (2007). Attribute differences between U.S. GAAP and IFRS earnings: an exploratory study. The International Journal of Accounting, 42, 123-142.

Ohlson, J. (1995). Earnings, book values, and dividends in equity valuation. Contemporary Accounting Research, 11(2), 661-687.

Phillips, J., Pincus, M., \& Rego, S. (2003). Earnings management: new evidence based on deferred tax expense. The Accounting Review, 78(2), 491-521.

Poterba, J., Rao, N., \& Seidman, J. (2011). Deferred tax positions and incentives for corporate behavior around corporate tax changes. National Tax Journal, 64(1), 27-58.

Schrand, C., \& Wong, M. (2003). Earnings management using the valuation allowance for deferred tax assets under SFAS No. 109. Contemporary Accounting Research, 20(3), 579-611.

Soderstrom, N., \& Sun, K. (2007). IFRS adoption and accounting quality: a review. European Accounting Review, 16(4), 675-702.

Wahab, N., \& Holland, K. (2012). Tax planning, corporate governance and equity value. The British Accounting Review, 44, 111-124.

Submetido: 13.04 .2014

Aceite: 23.10 .2014 